\title{
Prosthodontic Management of a Patient with Parkinson's Disease- A Case Report
}

\author{
K. Pavithra, Antonette Rhea, M. Dhanraj, Pravinya* \\ Department of Prosthodontics, Saveetha Dental College and Hospital, Saveetha Institute of Medical and Technical Sciences, Saveetha University, Chennai, Tamil Nadu, \\ INDIA
}

\begin{abstract}
Introduction: Parkinson's disease is a neurological disorder characterized by tremors, rigidity, bradykinesia and postural instability. Impairment of motor skills and cognition compromise the patient's diet, nutrition and ability to maintain proper oral hygiene. As a result dental caries and edentulism seem to be a direct impending predicament. It is important to understand that people with parkinson's disease start experiencing symptoms later in the course of the disease because a significant amount of the substantia nigra neurons have already been lost or impaired. Lewy bodies (accumulation of abnormal alphasynuclein) are found in substantia nigra neurons of PD patients. In addition to movement-related (motor) symptoms, Parkinson's symptoms may be unrelated to movement (non-motor). People with Parkinson's disease are often more impacted by their non-motor symptoms than motor symptoms such as apathy, depression, constipation, sleep behavior disorders, loss of sense of smell and cognitive impairment. Prosthodontics management of patients with parkinson's disease require diligent handling.Objectives and Methods: A patient with a history of Parkinsons disease for the past 5 years (under medication) visited Saveetha dental college with the chief complaint of broken prosthesis. High impact resin was chosen to be material of choice for this patient
\end{abstract}

due to its high flexural strength. So the prosthetic phase was modified and a drug delivery denture was fabricated to meet the demands of this patient. Results: Drug delivering partial denture was fabricated to meet the esthetic demands of the patient as well as to deliver the extended release formulation of the drug for managing the motor fluctuations.

Key words: Parkinson's disease, Tab. Pardopa, Drug delivery denture, High impact resin.

\section{Correspondence}

Antonette Rhea, Lecturer, Department of Prosthodontics, Saveetha Dental College and Hospital, Saveetha Institute of Medical and Technical Science, Saveetha University, 162, Poonamalle High Road, Velapanchavadi, Chennai -600077, Tamil Nadu, INDIA.

Phone: +91-9176645343

Email: arhea.a@gmail.com

DOI: 10.5530/jyp.2018.10.84

\section{INTRODUCTION}

Parkinson's disease (PD) is a long-term degenerative disorder of the central nervous system that mainly affects the motor system. The symptoms generally come on slowly over time. This is due to the progressive degeneration of nerve cells in the brain resulting in a decrease in dopamine levels. Dopamine is a chemical that helps in transmitting messages between cells. Most Parkinson's patients are old and are less likely to opt for dental treatments except for emergencies. ${ }^{1}$ Medication used to treat parkinsonism causes xerostomia which lead to increase in the risk of caries and fungal infections.

Early in the disease, the most obvious are shaking, rigidity, slowness of movement and difficulty with walking, thinking. Dementia becomes common in the advanced stages of the disease. ${ }^{2}$ Depression and anxiety are also common occurring in more than a third of people with Parkinson's disease. Other symptoms include sensory, sleep and emotional problems. The main motor symptoms are collectively called parkinsonian syndrome. The cause of Parkinson's disease is generally unknown but believed to involve both genetic and environmental factors. Those with a family member affected are more likely to get the disease themselves.

Removable partial dentures tend to be dislodged or swallowed while complete dentures fall out more often. They can even break following sudden jerky movements. If the person is a complete denture wearer then it becomes difficult to wear them, as the disease gets worse. ${ }^{3}$ Tremors caused by Parkinson's disease can make dental appointments a challenge. These patients have a hard time opening their mouth for longer time. Anxiety increases the Parkinson's symptoms. It is important that patient should remain calm during dental treatment. It is essential to make the environment stress free as possible. ${ }^{4}$ Here is one of such cases of a partially edentulous Parkinson's patient successfully treated with a drug delivery denture using auto-polymerising high impact resin.

\section{CASE REPORT}

A 55-year-old male patient reported to Saveetha dental college with a chief complaint of broken prosthesis and wanted replacement. The patient had a 5-year medical history of Parkinson's disease and was under medication. Patient was taking Tab. Pardopa twice daily for the past 5 years. Past dental history included fillings, extraction of teeth, and a removable partial prosthesis. He was able to communicate even though with some difficulty. He was well oriented, did not show signs of depression, and was highly motivated to replace his missing teeth. The intraoral examination revealed missing anterior teeth 11,21 and 12 (as seen in figure 4), dryness of the mouth. His physical and financial condition led to planning a denture with certain modifications.

Drug delivery denture is fabricated using auto-polymerising high impact resin. High impact resin was chosen to be the material of choice due to its high flexural strength. The treatment plan was finalized after consulting the patient's physician. The choice of drug used in denture was Tab. Pardopa (levodopa 100mg plus carbidopa $10 \mathrm{mg}$ ) manufactured by Synchro (Micro Labs Ltd) which is orally disintegrating and an extended release formulation. Pardopa Tablet contains carbidopa and levodopa as active ingredients and works by increasing the dopamine levels in the 
brain for proper brain functioning. Carbidopa allows more levodopa to enter the brain and is used for treating stiffness, tremors, spasms and poor muscle control in parkinson's disease and other related conditions. Levodopa has short half-life and therefore immediate release formulations lead to rapid increase and decrease in plasma levodopa concentration. Sustained release formulation helps in maintaining a steady plasma

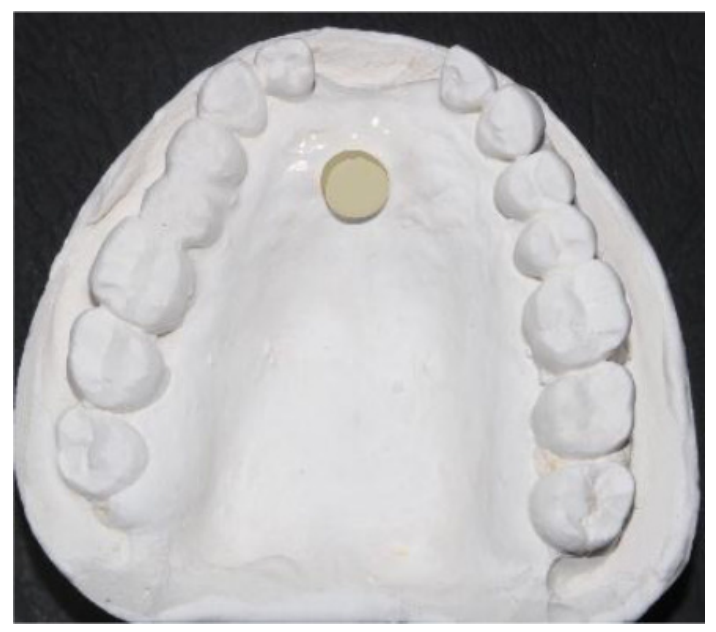

Figure 1: Primary Cast with Tablet Dimension.

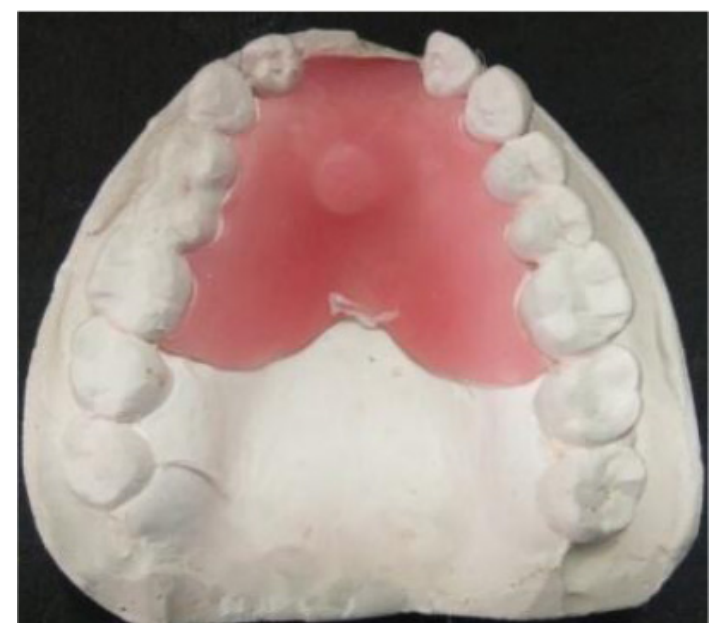

Figure 2: Denture base fabrication with slot for drug placement.

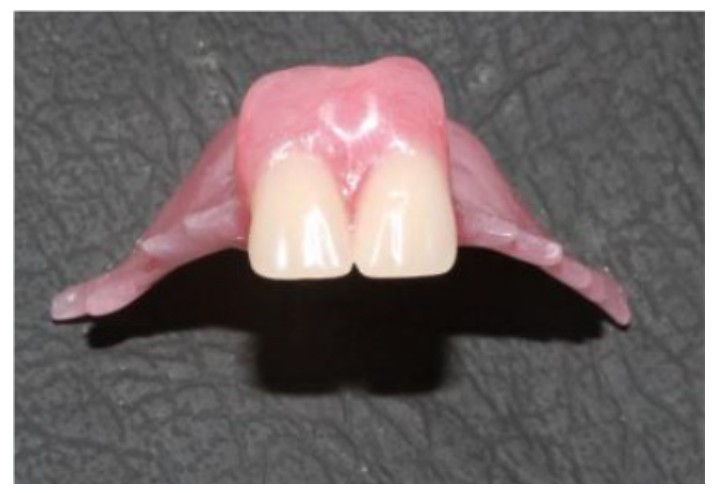

Figure 3: Final prosthesis.

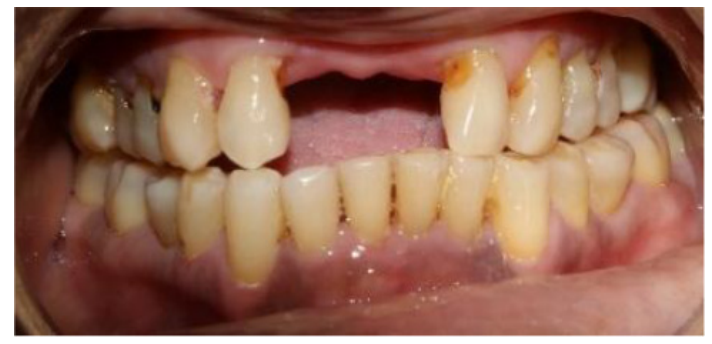

Figure 4: Pre-operative shows missing 11, 12 and 21.

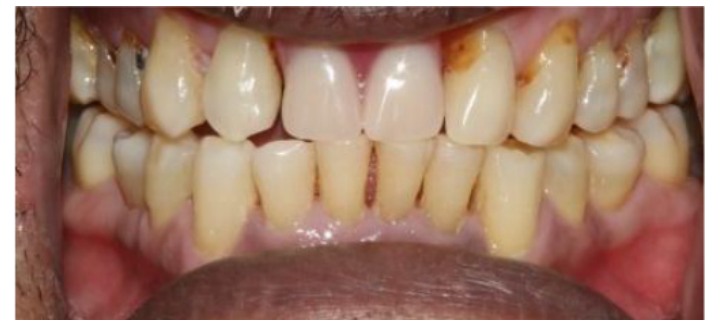

Figure 5: Post-operative shows replacement of missing teeth.

levodopa concentration and thus to manage the motor fluctuations. So, considering the advantages of extended release formulation of Pardopa over immediate release, a drug delivery denture was fabricated with a slot for placing the drug.

On the first appointment, primary impressions were made with Irreversible hydrocolloid i.e. alginate in the conventional manner using a stock tray and the primary cast was obtained (Figure 1$)$. Anterior teeth $(11,21)$ was selected based on patient's facial form (SR Vivadent from Ivoclar). An appropriate shade was chosen matching the adjacent teeth (A2 using Ivoclar vivadent shade guide).

Drug delivery slot was incorporated in the denture by placing the drug i.e. Tab. Pardopa in the anterior part of the palatal region. The primary cast obtained was coated with separating medium (DPI Cold mould seal).

The cast was left to dry and then auto-polymerising high impact resin (Lucitone HIPA from Dentsply) was used to fabricate the partial denture by sprinkle -on method (Figure 2). The tooth position was verified and after the final set of the acrylic, the partial denture with the slot created by placing the drug, was separated from the cast. The denture was trimmed of excess acrylic in a high speed dental lathe. The drug was removed and the drug delivering slot was refined by fine trimming and polished adequately. Finishing and polishing of the entire partial denture (as seen in Figure 3) was done to meet the esthetic demands of the patient.

\section{DISCUSSION}

Parkinson's disease is a chronic, progressive, neurodegenerative disorder, characterized by resting tremor (in hands, arms, legs, jaw and face), rigidity and stiffness (limbs and trunk), and postural instability or impaired balance and coordination. There are peculiar clinical features of this disease, like resting tremors, muscular rigidity and hypokinesia, facial impassiveness and cogwheel type of rigidity. ${ }^{5}$ This is due to insufficient formation and action of dopamine produced in dopaminergic neurons of mid brain. ${ }^{6}$ It is accompanied by various signs and symptoms, which affect the day-to-day activities of the patient. The physical symp- 
toms of Parkinson's disease present challenges for daily routine including dental care. ${ }^{7}$ Major component of oral hygiene and home care program requires muscle-eye coordination, digital dexterity, and tongue cheek-lip control. ${ }^{8}$ Tremor and the associated loss and/or reduction of the above faculties mitigate against effective oral hygiene procedures. Because of the poor motor function, nearly half of all people with parkinson's disease have difficulty with their daily oral hygiene regimen. For example, they are less likely than others in their age group to clean their dentures daily. Associated problems like rigidity and abnormal posture may make dental examination more difficult. ${ }^{9}$ Weakened swallowing ability can increase the risk of aspiration. ${ }^{10}$ Additionally, people with Parkinson's disease who have been on medication for several years begin to develop dyskinesias, which affects the jaw as well as teeth grinding both of which may create problems during dental treatment. ${ }^{11-12}$ People with Parkinson's disease also experience dry mouth, which contributes to or worsen already existing chewing difficulties or denture discomfort.

In addition to motor-related difficulties associated with Parkison's disease, there are additional behavioral changes that negatively affect dental care..$^{13}$ These include apathy, depression, and forgetfulness, all of which lead to negligence in daily oral health care. ${ }^{14-15}$ Dentists face many problems in fabrication of denture in such patients because increased tremors, increased saliva, diminished adaptive skills and poor muscle control make impression making and jaw relation recording difficult, causing compromised retention. ${ }^{16-17}$

The technique described here is relatively simple and a drastic departure from the conventional procedure. ${ }^{18}$ With this technique, denture was delivered in two visits and was also very economical for the patient. Although this technique increases laboratory time, it reduces the clinical visits to a greater extent without compromising the basic principles of denture fabricated.

\section{CONCLUSION}

The psychological and behavioral pattern associated Parkinson's disease can cause major difficulties during the fabrication of a dental prosthesis. ${ }^{19}$ The success of the prosthesis will depend on the careful approach with diligent handling of the patient during the entire therapy. Drug delivering partial denture replacing 11,21 was thus fabricated (as seen in figure 5) to meet the esthetic demands of the patient as well as to deliver the extended release form of Pardopa tablet for managing the motor fluctuations. ${ }^{20}$ Educating the patient and his family regarding the post insertion care of the prosthesis is essential for the long term success of the treatment.

\section{REFERENCES}

1. De Lau LM, Breteler MM. Epidemiology of Parkinson's disease. Lancet Neurol. 2006;5(6):525-35.

2. Davie CA. A review of Parkinson's disease. Br Med Bull. 2008;86(1):109-27.

3. Jankovic J. Parkinson's disease: Clinical features and diagnosis. J Neurol Neuro- surg Psychiatry. 2008;79(4):368-76.

4. Tzen KY, Lu CS, Yen TC, Wey SP, Ting G. Differential diagnosis of Parkinson's disease and vascular parkinsonism by 99mTc-TRODAT-1. J Nucl Med. 2001; 42(3):408-13.

5. National Collaborating Centre for Chronic Conditions. Symptomatic pharmacological therapy in Parkinson's disease. Parkinson's disease. London: Royal College of Physicians. 2006:59-100.

6. Hasnain M, Vieweg WV, Baron MS, Beatty-Brooks M, Fernandez A, Pandurangi AK. Pharmacological management of psychosis in elderly patients with Parkinsonism. Am J Med. 2009;122(7):614-22.

7. Lee MS, Lam P, Ernst E. Effectiveness of tai chi for Parkinson's disease: A critical review. Parkinsonism and Related Disorders. 2008;14(8):589-94.

8. Friedlander $\mathrm{AH}$, Mahler $\mathrm{M}$, Norman KM, Ettinger RL. Parkinson disease: Systemic and orofacial manifestations, medical and dental management. J Am Dent Assoc. 2009;140(6):658-69.

9. Bhat V, Prasad K, Balaji SS, Bhat A. Complete denture treatment protocol in Parkinson's disease. A case report. J Ind Acad Dent Spec. 2011;2(2):63-5.

10. Walker BR, Colledge NR. Davidson's Principles and Practice of Medicine E-Book. Elsevier Health Sciences. 2013.

11. Persson M, Sterberg TO, Granérus AK, Karlsson S. Influence of Parkinson's disease on oral health. Acta Odontol Scand. 1992;50(1):37-42.

12. Chu FC, Deng FL, Siu AS, Chow TW. Implant-tissue supported, magnet-retained mandibular overdenture for an edentulous patient with Parkinson's disease: A clinical report. J Prosthet Dent. 2004;91(3):219-22.

13. Packer $M$, Nikitin $V$, Coward T, Davis DM, Fiske J. The potential benefits of dental implants on the oral health quality of life of people with Parkinson's disease. Gerodontology. 2009;26(1):11-8.

14. Rajeswari CL. Prosthodontic considerations in Parkinson's disease. PJSR. 2010;3:45-47.

15. Ansari IH. A one-appointment impression and centric relation record technique for compromised complete denture patients. J Prosthet Dent. 1997;78(3):320-3.

16. Friedlander $\mathrm{AH}$, Mahler $\mathrm{M}$, Norman KM, Ettinger RL. Parkinson disease: Systemic and orofacial manifestations, medical and dental management. J Am Dent Assoc. 2009;140(6):658-69.

17. Langer A. Prosthodontic failures in patients with systemic disorders. J Oral Rehabil. 1979;6(1):13-9.

18. Kieser J, Jones G, Borlase G, MacFadyen E. Dental treatment of patients with neurodegenerative disease. N Z Dent J. 1999;95(5):130-4.

19. Monalisa R. Incidence of adverse effects of dental materials on dentist's survey based questionnaire. Res J of Pharm Technol. 2016;9(10):1599-601.

20. Friedlander $\mathrm{AH}$, Mahler $\mathrm{M}$, Norman KM, Ettinger RL. Parkinson disease: Systemic and orofacial manifestations, medical and dental management. J Am Dent Assoc. 2009;140(6):658-69.

21. Deepa Shenoy, K.M. Mathew, Ashish Jain. Evaluation of Impact and flexural strength of conventional Heat cure Polymethyl methacrylate resin and High Impact. Biol Med. 2016;9(2):2-4.

22. Charan VS. Etiology, Treatment and Management of Parkinson's - A Review. J Pharm Sci Res. 2016;8(8):754-55.

23. Subramanian A, Muthukumaran S. Rapid preparation process of antiparkinsonian drug Mucuna pruriens silver nanoparticle. Pharmacognosy Res. 2010;2(4):233-6

24. Anil S, H Jain, Vipin S, Edwin JE Ahmad Showkat Colon-targeted quercetin delivery using natural polymer to enhance its bioavailability. Pharmacognosy Res. $2011 ; 3(1): 35-9$

Article History: Submission Date : 20-04-2018; Revised Date : 23-05-2018; Acceptance Date : 22-06-2018.

Cite this article: Pavithra K, Dhanraj M, Pravinya, Rhea A. Prosthodontic Management of a Patient with Parkinson's disease- A Case Report. J Young Pharm. 2018;10(3):377-9 